\title{
Extended use of the modified Berlin Definition based on age- related subgroup analysis in pediatric ARDS
}

\author{
Michael Hermon · Sophia Dotzler · Jennifer Bettina Brandt · Wolfgang Strohmaier · Johann Golej
}

Received: 23 May 2018 / Accepted: 16 August 2018 / Published online: 19 September 2018

(c) The Author(s) 2018

\begin{abstract}
Summary
Background Pediatric acute respiratory distress syndrome (pARDS) is a rare but very severe condition. Management of the condition remains a major challenge for pediatric intensive care specialists.

Objective To perform a descriptive assessment of pARDS based on the modified Berlin Definition by using the $\mathrm{SpO}_{2} / \mathrm{FiO}_{2}$ ratio in order to establish an extended patient registry divided into age-related subgroups.
\end{abstract}

\footnotetext{
Authors' contributions and statement MH conceived the study and participated in the design and execution of the study, the analysis of data, and writing of the manuscript. SD participated in the design and execution of the study, and performed data collection, statistical analysis, and interpretation of the data. JB performed data collection and was responsible for the graphic design of the tables and figures. WS participated in the interpretation of results and writing the manuscript. JG supervised the study and is program director. All authors read and approved the final manuscript.
}

M. Hermon, MD $(\varangle) \cdot$ J. B. Brandt · J. Golej

Division of Neonatology, Pediatric Intensive Care and Neuropediatrics, Medical University of Vienna, Währinger Gürtel 18-20, 1090 Vienna, Austria

michael.hermon@meduniwien.ac.at

J. B. Brandt

jennifer.brandt@meduniwien.ac.at

J. Golej

johann.golej@meduniwien.ac.at

S. Dotzler

Wilheminenspital, Vienna, Austria

sophiadotzler@hotmail.com

W. Strohmaier

Institut für molekulare Biowissenschaften, Karl Franzens

University Graz, Graz, Austria

w.strohmaier@chello.at
Methods The data of all children on mechanical ventilation for respiratory failure admitted between 2005 and 2012 were reviewed retrospectively for this study. The age of patients ranged from newborns $>37$ weeks, up to children $<18$ years. Inclusion criteria were based on the modified Berlin Definition of pARDS. The following data were collected: demographic data, primary diagnosis, ventilation settings, and use of supportive treatment, in addition to mechanical ventilation (inhaled nitric oxide, surfactant, corticosteroids, prone positioning, and extracorporeal membrane oxygenation).

Results In all, 93 children where included: $35 \%$ were newborns, $29 \%$ infants, $24 \%$ toddlers, and $12 \%$ school children; $66 \%$ were male and $34 \%$ were female patients. The most common primary diagnosis was viral pneumonia $(21 \%)$ and $55 \%$ of the children were diagnosed with severe ARDS. The median duration of stay on the pediatric intensive care unit was 16 days (10/27). In total, 66 children (71\%) had direct lung injury and 18 (19\%) had indirect lung injury. More than $80 \%$ of all children needed more than one supportive care therapy. The overall survival rate was $77 \%$.

Conclusion This study is a valuable report about pediatric patients with ARDS and allows for an important extension of the application of the modified Berlin Definition in all age groups.

Keywords Pediatric ARDS - Children - Berlin Definition - ARDS supportive treatment $\cdot \mathrm{SpO} 2 / \mathrm{FiO} 2$ ratio
Abbreviation
ARDS acute respiratory distress syndrome
pARDS pediatric acute respiratory distress syndrome
AECC American-European Consensus Conference
BD Berlin Definition
ECMO extracorporeal membrane oxygenation
iNO inhaled nitric oxide 
PICU pediatric intensive care unit

$\mathrm{PaO}_{2}$ arterial partial pressure of oxygen

$\mathrm{SpO}_{2}$ peripheral oxygen saturation

$\mathrm{FiO}_{2}$ fractional inspired oxygen concentration

MV mechanical ventilation

CHD congenital heart defect

\section{Introduction}

Acute respiratory distress syndrome (ARDS), as first described by Ashbaugh et al. in 1967 [1], remains a therapeutical challenge for intensive care specialists. Pediatric ARDS (pARDS) can be described as a rare disease with an incidence of 2-12/100,000 per year. The low number of patients with pARDS makes it a challenging task to conduct clinical trails with conclusive results [2,3]. At present, the overall mortality rate in pARDS is approximately $24 \%$ [4]. Over many years, pediatric intensive care specialists have used the American-European Consensus Conference (AECC) definition of ARDS for clinical care, research, and prognosis [5]. Limitations of the AECC definition of ARDS have recently been addressed by the Berlin Definition (BD) for adult patients, but pediatric-specific considerations were not included in these definitions $[5,6]$. Although there are similarities in the pathophysiology of ARDS in adults and children, pediatric-specific therapies, comorbidities, and differences in outcome demand a more specific definition for pARDS patients [7]. In this context, a major limitation of the adult $\mathrm{BD}$ is the necessity for invasive measurement of arterial blood gas. Pulse oximetry increasingly prevents the use of arterial blood gas measurement in children, and therefore a definition requiring direct blood gas analysis may underestimate the prevalence of pARDS. Moreover, recent evidence shows that arterial catheters are an under-recognized source of infection and this may continue to contribute to a shift in practice patterns away from the routine use of such arterial catheters [8] and consequently $\mathrm{PaO}_{2}$ measurements. Several studies have demonstrated that the $\mathrm{SpO}_{2} / \mathrm{FiO}_{2}$ ratio (S/F ratio) can be used instead of the $\mathrm{PaO}_{2} / \mathrm{FiO}_{2}(\mathrm{P} / \mathrm{F}$ ratio) when the $\mathrm{SpO}_{2}$ is $<97 \%$ [9-12].

In October 2013, De Luca et al. published a multicenter study with the main goal of investigating the validity of BD in infancy and early childhood [13]. Our study attempts to extend the systematical data collection and provide a more detailed description of pARDS patients divided into age-related subgroups (full-term newborns 0-28 days, infants 1-12 months, children 1-6 years, children 6-18 years). ARDS etiology (direct and indirect lung injury), severity of ARDS according to the modified $\mathrm{BD}$, and five types of supportive treatment options were also investigated: inhaled nitric oxide (iNO), surfactant, corticosteroids, prone position, and extracorporeal membrane oxygenation (ECMO).
The objective of this study was to describe a cohort of patients with pARDS using the modified BD with the S/F ratio in order to establish an extended patient registry divided into age-related subgroups. A further aim of this study was to provide feedback to clinicians about their daily work concerning the quality of treatment and possible options for the improvement of treatment in the future.

\section{Patients and methods}

After the approval of the ethics committee (Medical University of Vienna; Ethic Nr. 1860/2012) the patient records of all children admitted to our pediatric intensive care unit (PICU) between 2005 and 2012 were screened for eligibility. Because of the retrospective character of this study, informed consent was waived and the study was performed in accordance with the Declaration of Helsinki.

The data of 219 children-aged between full-term newborns and $<18$ years-with respiratory failure according to the modified BD of ARDS [6] were reviewed for inclusion in the study. Only patients with complete records were included. The 93 patients finally included were divided into three groups according to the ARDS etiology, direct and indirect lung injury, and a third group of ARDS patients with unknown etiology. Preterm newborns with a corrected age of $>38$ weeks of gestation at the time of admission were not excluded from the study. The following data were extracted from patient files: age (years), gender, weight $(\mathrm{kg})$, duration of PICU stay (days). Primary diagnosis, relevant secondary diagnosis, and chronic or congenital diseases or developmental disorders were noted. The modes and duration of mechanical ventilation (MV) and ventilator settings were recorded. Oxygenation was evaluated by recording arterial blood gas samples and $\mathrm{SpO}_{2}$. If arterial blood gas samples were not available, the $\mathrm{S} / \mathrm{F}$ ratio was used as a surrogate for P/F ratio [10-12]. Additional supportive treatment beside MV, such as iNO, surfactant, corticosteroids, prone positioning, and ECMO were noted. Outcomes were defined as survival of PICU stay.

Chest radiographs and levels of oxygenation were collected on day 1, day 3, and day 7 of PICU stay. However, these radiographs were not available for all patients for each of the 3 days, and thus patients were included if at least one chest radiograph was available. The chest radiographs were anonymized and examined by an independent radiologist.

Data analysis was performed using IBM SPSS Statistics Version 21.0 (IBM Deutschland GmbH, Ehningen, Germany). For statistical analysis, the chi-square test or Fisher's exact test and independent-samples Kruskal-Wallis test were used. Results were accepted as statistically significant at $p<0.05$. Data are presented as median and interquartile ranges or as percentages. 


\section{Results}

During the study period 2005-2012, 219 patient records were screened for eligibility criteria and 93 children were included in the study. We excluded 126 patients owing to missing data or chest radiographs. Table 1 shows the demographic data of all study patients. Because of the heterogeneity of the patient collective with a range in age from 0 days to 16.8 years, patients were divided into age-related subgroups to account for age-specific conditions and differences. The subgroup of school children was not divided any further owing to the small number of patients of this age. Seven patients $(7 \%)$ had a history of preterm birth but had reached a corrected age of $>38$ weeks of gestation at admission and therefore were included.

Table 1 Demographic data of study patients

\begin{tabular}{|l|l|l}
\hline Total number of patients; $N(\%)$ & $93(100)$ & - \\
\hline $\begin{array}{l}\text { Age in years; median (IQR) } \\
\text { Gender: M/F; } N \text { (\%) }\end{array}$ & $0.3(0-16.8)$ & - \\
\hline History of preterm birth; $N(\%)$ & $71(66) / 32(34)$ & - \\
\hline $\begin{array}{l}\text { PICU stay in days; median (IQR) } \\
\text { Duration of mechanical ventilation; } \\
\text { median (IQR) }\end{array}$ & $16(10-27)$ & - \\
\hline Survival of PICU stay; $N(\%)$ & $72(77)$ & - \\
\hline Age-related subgroups & $N(\%)$ & - \\
\hline Newborns (0-28 days) & $33(35)$ & $22 / 11$ \\
\hline Infants (1-12 months) & $27(29)$ & $21 / 6$ \\
\hline Children (1-6 years) & $22(24)$ & $12 / 10$ \\
\hline Children (6-18 years) & $11(12)$ & $6 / 5$ \\
\hline IQR interquartile range, Ffemale, $M$ male PICUpediatric intensive care unit
\end{tabular}

Table 2 Etiologies of pARDS

\begin{tabular}{|c|c|c|c|c|c|c|c|c|}
\hline \multirow[t]{2}{*}{ Diagnosis } & \multicolumn{2}{|c|}{$\begin{array}{l}\text { Newborn } \\
(0-28 d)\end{array}$} & \multicolumn{2}{|c|}{$\begin{array}{l}\text { Infant } \\
(1-12 \mathrm{mo})\end{array}$} & \multicolumn{2}{|c|}{$\begin{array}{l}\text { Children } \\
(1-6 y)\end{array}$} & \multicolumn{2}{|c|}{$\begin{array}{l}\text { Children } \\
\text { (6-18 y) }\end{array}$} \\
\hline & $(M)$ & $(\%)$ & $(M)$ & $(\%)$ & $(M)$ & $(\%)$ & $(M)$ & $(\%)$ \\
\hline MAS & 10 & 31 & - & & - & & - & \\
\hline $\mathrm{CDH}$ & 6 & 18 & - & & - & & - & \\
\hline Sepsis & 5 & 15 & 3 & 11 & 3 & 14 & 1 & 9 \\
\hline $\begin{array}{l}\text { Respiratory fail- } \\
\text { ure not specified }\end{array}$ & 5 & 15 & 4 & 15 & 5 & 23 & 1 & 9 \\
\hline $\begin{array}{l}\text { Bacterial pneu- } \\
\text { monia }\end{array}$ & 2 & 6 & 4 & 15 & 4 & 18 & 4 & 37 \\
\hline Viral pneumonia & - & & 14 & 51 & 5 & 23 & 1 & 9 \\
\hline CHD & 2 & 6 & - & & 1 & 4 & 1 & 9 \\
\hline Lung bleeding & 1 & 3 & 1 & 4 & - & & - & \\
\hline $\begin{array}{l}\text { Alveolar pro- } \\
\text { teinosis }\end{array}$ & 1 & 3 & - & & - & & 1 & 9 \\
\hline Asphyxia & 1 & 3 & - & & - & & - & \\
\hline $\mathrm{CF}$ & - & & 1 & 4 & 1 & 4 & 1 & 9 \\
\hline Near drowning & - & & - & & 3 & 14 & 1 & 9 \\
\hline Total & 33 & 100 & 27 & 100 & 22 & 100 & 11 & 100 \\
\hline
\end{tabular}

Table 1 also shows the number of patients stratified into age-related subgroups and gender. There were more male patients in the age groups of newborns and infants, but the gender distribution between age-related subgroups was not significantly different $(p>0.05)$. There was an overall majority of males in this study and no significant difference of survival was recorded between male and female patients $(77 \%$ vs. $78 \% ; p>0.05$ ).

The median duration of PICU stay was 16 (10-27) days. In total, 66 patients $(71 \%)$ had direct lung injury, 18 patients (19\%) had indirect lung injury, and nine patients (10\%) had lung injuries of unknown origin. No significant difference was found between the age-related subgroups concerning type of lung injury. Survival of patients with direct lung injury was $80 \%$ vs. $67 \%$ in patients with indirect lung injury. The survival of patients with direct lung injury was higher, but not significant $(p>0.05)$. The most common diagnosis leading to pARDS was viral pneumonia $(21 \%)$, followed by nonspecified respiratory failure (16\%) and bacterial pneumonia (15\%). In $4 \%$ of all cases, the primary diagnosis was congenital heart defect (CHD). These patients developed pARDS additionally and secondary to their primary diagnosis of CHD during their PICU stay. The etiology of pARDS within the age-related subgroups is shown in Table 2. Viral pneumonia was among the most common causes of pARDS in infants $(51 \%)$ and toddlers $(23 \%)$. In children older than 6 years, bacterial pneumonia was the most common cause of pARDS with four patients (36\%) presenting this diagnosis. The severity of pARDS according to $\mathrm{BD}$ on the day of admission is shown in Fig. 1. There was no significant difference in the survival rate or the duration of MV according to the severity of ARDS. Severity

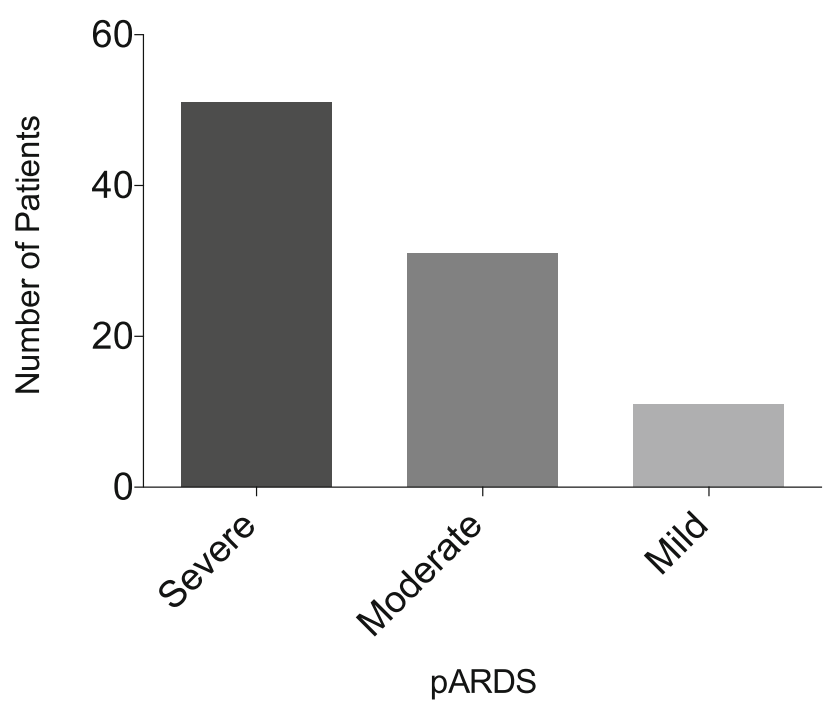

Fig. 1 Severity of pediatric acute respiratory distress syndrome ( $p A R D S)$. According to the modified Berlin Definition, most of the patients had severe pARDS on the day of admission 
Table 3 Supportive therapy options ${ }^{\mathrm{a}}$

\begin{tabular}{l|l|l|l|} 
Supportive therapy & $N(\%)$ & $\begin{array}{l}\text { Therapy } \\
\text { options }\end{array}$ & $N(\%)$ \\
\hline Corticosteroids & $71(76)$ & None & $8(9)$ \\
\hline iNO & $55(59)$ & One & $10(11)$ \\
\hline Prone position & $50(54)$ & Two & $21(23)$ \\
\hline Surfactant & $49(53)$ & Three & $27(29)$ \\
\hline ECMO & $23(27)$ & Four & $20(21)$ \\
\hline & & Five & $7(7)$
\end{tabular}

iNO inhaled nitric oxide, ECMO extracorporeal membrane oxygenation ${ }^{a}$ Most of the patients $(N=76,76 \%)$ received corticosteroids as supportive therapy beside mechanical ventilation. About every second patients was placed in prone position and treated with surfactant. Most of the patients had three different supportive therapies

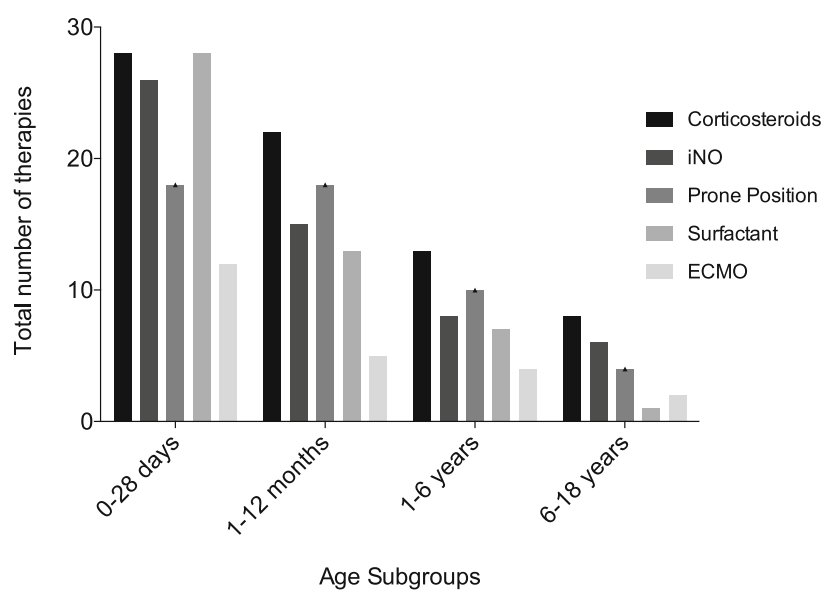

Fig. 2 Supportive care treatment separated into age-related subgroups. The figure shows the distribution of treatment strategies between the four age subgroups. The distribution refers to the total number of the supportive therapies that were used and not to the number of patients. ECMO extracorporeal membrane oxygenation, iNO inhaled nitric oxide

was also not significantly different among age-related subgroups $(p>0.05)$.

There were significant differences in the mode of ventilation used between the age-related subgroups $(p<0.001)$. Pressure-controlled ventilation was used in all age-related subgroups and was the leading mode of MV in patients older than 1 month. Noninvasive ventilation was used in two newborns and one school child. The median duration of MV was 12 days (7-22). The duration of MV was not significantly different among age-related groups.

Five types of supportive therapy options were investigated: iNO, surfactant, corticosteroids, prone positioning, and ECMO. Of all the patients, $75(80 \%)$ received more than such treatment option. An overview of additional supportive treatments is listed in Table 3. A combination of three types of supportive therapies was most frequently applied to patients $(n=27,29 \%)$. Eight patients $(9 \%)$ received nonsupportive therapy beside MV and seven patients $(7 \%)$ received all five supportive therapies. The impact of each single type of therapy was not analyzed separately. Despite the large number of different combinations of supportive treatment options, the combination of iNO, surfactant, corticosteroids, and prone positioning was the most common combination-used in 13 cases. Table 3 shows the overall frequency of supportive therapy applied to the study patients. The distribution of treatment strategies between the age-related subgroups is shown in Fig. 2. In total, $81 \%$ of all patients received more than one supportive therapy, and thus the distribution refers to the total number of supportive therapies used (and not to the number of patients). ECMO was used in 23 children, most of whom were $<1$ year. The median duration of ECMO was 11 days (7-16). The overall survival rate of patients who received ECMO was $52 \%$.

\section{Discussion}

This study evaluated pARDS patients and additional supportive treatments by using an age-related subgroup analysis. The first finding is that $66 \%$ of the 93 patients enrolled were male. This strong predominance of male gender was surprising since to date ARDS has not been reported to affect males more than females. Several studies on pARDS have shown male predominance but less pronounced-from 54 to $59 \%$ $[2,3,14]$.

Patients were more frequently diagnosed with direct lung injury (71\%) than indirect lung injury (19\%). Patients with direct lung injury had an $80 \%$ survival rate, while the rate for indirect lung injury patients was $67 \%$. Survival rates were not significantly different between these groups. This finding is in line with earlier pARDS studies, where the lowest survival rates were associated with sepsis, which is an indirect etiology of lung injury [14].

In this study, infection was the most common direct lung injury and viral pneumonia the most frequent diagnosis overall. Stratification by age subgroups showed that viral pneumonia is the most common diagnosis in infants (1-12 months) and among children (1-6 years), while children older than 6 years where affected by bacterial pneumonia. This finding confirms an earlier report by Wainwright [15].

The enrollment of newborns to pARDS studies is worthy of ongoing discussion. This period of life is characterized by a high risk of mortality [16], specifically among preterm newborns. Primary RDS related to prematurity is clearly different from pARDS in many aspects and consequently these patients were excluded. In full-term newborns, age specific-etiologies such as meconium aspiration and congenital diaphragmatic hernia serve as triggers for the development of pARDS. There is no clinical or biological evidence suggesting that lung injury in this group differs from older pARDS patients [17]. Since almost $36 \%$ of our patient collective were newborns and had pARDS, the management of these patients in our PICU plays an important role. Therefore, we decided to include 
full-term newborns like the other age groups into this study.

The severity of ARDS was defined according to BD. De Luca et al. reported that BD has improved validity for pARDS patients compared with the old AECC definition [13]. In this study, however, no significant differences were found in PICU survival or duration of MV measures. One reason might be the smaller number of patients compared with those in the study of De Luca et al., who analyzed 221 children in their multicenter study. In addition, the very heterogeneous patient collective presented here (patients ranged from newborns to children $<18$ years) differs from the study of De Luca et al., which focused on infants and toddlers aged $>30$ days and $<18$ months. Furthermore, the duration of MV was analyzed for all patients including those who did not survive. This might bias toward a shorter duration of MV (due to severely ill patients who died early in the course of treatment while being mechanically ventilated). The validity of this study's results may be limited owing to the retrospective data collection and because levels of oxygenation were only collected at selected time points: namely, the day of admission, day 3 , and day 7 of PICU stay.

To classify the severity of pARDS, the lowest oxygenation level of the admission day was used. Since most patients did not have arterial blood samples taken, the $\mathrm{S} / \mathrm{F}$ ratio was used instead of the $\mathrm{P} / \mathrm{F}$ ratio, according to the regression equation $1 / \mathrm{SF}=0.00232+$ $0.443 / \mathrm{PF}$ demonstrated by Khemani et al. This was used as a valuable surrogate for $\mathrm{SpO}_{2}$ levels between 80 and $97 \%$ [11]. All 93 patients included in the study were within this range.

The majority of this study's patients received more than one supportive therapy. This explains why the impact of a single supportive therapy could not be analyzed. Furthermore, in this heterogeneous patient collective, those who did not receive one particular treatment would work poorly as a control group-the patient characteristics between the two patient groups are likely to show strong variety. However, one purpose of this study is its use as a basis for further prospective data collection where these limitations can be avoided.

The findings of the beneficial effects of the supportive treatment strategies investigated in this study are controversial. Although corticosteroids have been shown to improve the outcome of patients with meconium aspiration syndrome, data on the use of corticosteroids in pARDS are very limited [18]. Nevertheless, it was found to be the most frequently used supportive therapy in this study (76\%). iNO was used in $59 \%$ of all our patients. The use of iNO has been reported to transiently improve oxygenation without improving patient outcome. iNO might be more effective in patients with very severe oxygenation deficit and in immunocompromised patients [19, 20]. Results concerning prone positioning showed improved oxygenation in children and adults with a tendency to lead to a survival benefit in patients with severe oxygenation deficit in adult studies [21, 22]. Prone positioning was the third most common supportive therapy (54\%) for patients in this study. A recently published study shows that the response to prone positioning was variable in children with pARDS. Prone positioning improves the homogeneity of ventilation and may enhance recruitment of the dorsal lung regions [23].

More than $50 \%$ of patients in this study received surfactant as a supportive therapy; most of these patients were full-term newborns or infants. Similar findings are also reported in two studies where surfactant application was recommended especially in newborns [18, 24]. ECMO was also more frequently performed in newborns, showing a declining frequency of use with age. According to the ELSO Registry Report of 2016, the incidence of ECMO support has increased significantly in pediatric patients in general [25]. Recently published information on the ECMO survival rate of pediatric patients based on ELSO registry data showed a rate of $57 \%$ - slightly higher than the ECMO survival rate of $52 \%$ noted in this study [25]. However, in seven of the 23 ECMO patients, renal failure was present-something that has been reported as a contributing factor to reduced ECMO survival [26].

This study represents a valuable report on pediatric patients with ARDS and allows for an important extension of the application of the modified BD in all age groups. Moreover, this report suggests that the impact of therapeutic supportive therapies can be improved in the future by prospective data collection.

Funding No specific funding was received for this study.

Funding Open access funding provided by Medical University of Vienna.

\section{Compliance with ethical guidelines}

Conflict of interest M. Hermon, S. Dotzler, J.B. Brandt, W. Strohmaier, and J. Golej declare that they have no competing interests and financial potential conflicts do not exist.

Ethical standards All procedures performed in studies involving human participants were in accordance with the ethical standards of the institutional and/or national research committee and with the 1964 Helsinki declaration and its later amendments, or comparable ethical standards. For this type of study (retrospective study) informed consent is not required. This study was done with the approval of the of the ethics committee (Medical University of Vienna; Ethic Nr.1860/2012).

Open Access This article is distributed under the terms of the Creative Commons Attribution 4.0 International License (http://creativecommons.org/licenses/by/4.0/), which permits unrestricted use, distribution, and reproduction in any medium, provided you give appropriate credit to the original author(s) and the source, provide a link to the Creative Commons license, and indicate if changes were made. 


\section{References}

1. Ashbaugh DG, Bigelow DB, Petty TL, Levine BE. Acute respiratory distress in adults. Lancet. 1967;2(7511):319-23.

2. Zimmerman JJ, Akhtar SR, Caldwell E, Rubenfeld GD. Incidence and outcomes of pediatric acute lung injury. Pediatrics. 2009;124(1):87-95. https://doi.org/10.1542/ peds.2007-2462.

3. Kneyber MCJ, Brouwers AGA, Caris JA, Chedamni S, Plötz FB. Acute respiratory distress syndrome: is it underrecognized in the pediatric intensive care unit? intensive Care Med. 2008;34(4):751-4. https://doi.org/10.1007/s00134008-1029-4.

4. Wong JJ-M, Jit M, Sultana R, et al. Mortality in pediatric acute respiratory distress syndrome: a systematic review and Meta-analysis. J Intensive Care Med. 2017; https:/ / doi. org/10.1177/0885066617705109.

5. Bernard GR, Artigas A, Brigham KL, The American-European Consensus Conference on ARDS, et al. Definitions, mechanisms, relevant outcomes, and clinical trial coordination. Am J Respir Crit Care Med. 1994;149(3 Pt 1):818-24. https://doi.org/10.1164/ajrccm.149.3.7509706.

6. ARDS Definition Task Force, Ranieri VM, Rubenfeld GD, et al. Acute respiratory distress syndrome: the Berlin Definition. JAMA. 2012;307(23):2526-33. https://doi.org/ $10.1001 /$ jama.2012.5669.

7. Pediatric Acute Lung Injury Consensus Conference Group. Pediatric acute respiratory distress syndrome: consensus recommendations from the Pediatric Acute Lung Injury Consensus Conference. Pediatr Crit Care Med. 2015;16(5):428-39. https://doi.org/10.1097/PCC. 0000000000000350.

8. O'Horo JC, Maki DG, Krupp AE, Safdar N. Arterial catheters as a source of bloodstream infection: a systematic review and meta-analysis. Crit Care Med. 2014;42(6):1334-9. https://doi.org/10.1097/CCM.0000000000000166.

9. Thomas NJ, Shaffer ML, Willson DF, Shih M-C, Curley MAQ. Defining acute lung disease in children with the oxygenation saturation index. Pediatr Crit Care Med. 2010;11(1):12-7. https://doi.org/10.1097/PCC. 0b013e3181b0653d.

10. Khemani RG, Patel NR, Bart RD, Newth CJL. Comparison of the pulse oximetric saturation/fraction of inspired oxygen ratio and the $\mathrm{PaO} 2 /$ fraction of inspired oxygen ratio in children. Chest. 2009;135(3):662-8. https://doi.org/10. 1378/chest.08-2239.

11. Khemani RG, Thomas NJ, Venkatachalam V, et al. Comparison of $\mathrm{SpO} 2$ to $\mathrm{PaO} 2$ based markers of lung disease severity for children with acute lung injury. Crit Care Med. 2012;40(4):1309-16. https://doi.org/10.1097/CCM. 0b013e31823bc61b.

12. Mayordomo-Colunga J, Pons M, López Y, et al. Predicting non-invasive ventilation failure in children from the $\mathrm{SpO}_{2} / \mathrm{FiO}_{2}$ (SF) ratio. intensive Care Med. 2013;39(6):1095-103. https://doi.org/10.1007/s00134013-2880-5.

13. De Luca D, Piastra M, Chidini G, et al. The use of the Berlin definition for acute respiratory distress syndrome during infancy and early childhood: multicen- ter evaluation and expert consensus. intensive Care Med. 2013;39(12):2083-91. https://doi.org/10.1007/ s00134-013-3110- $\mathrm{x}$.

14. Flori HR, Glidden DV, Rutherford GW, Matthay MA. Pediatric acute lung injury: prospective evaluation of risk factors associated with mortality. Am J Respir Crit Care Med. 2005;171(9):995-1001. https://doi.org/10.1164/ rccm.200404-544OC.

15. Wainwright $C$. Acute viral bronchiolitis in children-a very common condition with few therapeutic options. Paediatr Respir Rev. 2010;11(1):39-45. https://doi.org/10.1016/j. prrv.2009.10.001.

16. WHO. Infant, Newborn. 2017. http://www.who.int/topics/ infant newborn/en/. Accessed26Mar 2017.

17. De Luca D, van Kaam AH, Tingay DG, et al. The Montreux definition of neonatal ARDS: biological and clinical background behind the description of a new entity. Lancet Respir Med. 2017;5(8):657-66.

18. DeLucaD, Piastra M, Tosi F, et al. Pharmacological therapies for pediatric and neonatal ALI/ARDS: an evidence-based review. Curr Drug Targets. 2012;13(7):906-16.

19. Afshari A, Brok J, Møller AM, Wetterslev J. Inhaled nitric oxide for acute respiratory distress syndrome (ARDS) and acute lung injury in children and adults. Cochrane Database Syst Rev. 2010;7:CD2787. https://doi.org/10. 1002/14651858.CD002787.pub2.

20. Abroug F, Ouanes-Besbes L, Dachraoui F, Ouanes I, Brochard L. An updated study-level meta-analysis of randomised controlled trials on proning in ARDS and acute lung injury. Crit Care. 2011;15(1):R6. https://doi.org/10. 1186/cc9403.

21. Gillies D, Wells D, BhandariAP. Positioning for acuterespiratory distress in hospitalised infants and children. Cochrane Database Syst Rev. 2012;7:CD3645. https://doi.org/10. 1002/14651858.CD003645.pub3.

22. Curley MAQ, Hibberd PL, Fineman LD, et al. Effect of prone positioning on clinical outcomes in children with acute lung injury: a randomized controlled trial. JAMA. 2005;294(2):229-37. https://doi.org/10.1001/jama.294.2. 229.

23. Lupton-Smith A, Argent A, Rimensberger P, Frerichs I, MorrowB.Pronepositioningimprovesventilationhomogeneity in children with acute respiratory distress syndrome. Pediatr Crit Care Med. 2017;18(5):e229-e34. https://doi.org/10. 1097/PCC.0000000000001145.

24. Duffett M, Choong K, Ng V, Randolph A, CookDJ. Surfactant therapy for acute respiratory failure in children: a systematic review and meta-analysis. Crit Care. 2007;11(3):R66. https://doi.org/10.1186/cc5944.

25. Barbaro RP, Paden ML, GunerYS, et al. Pediatric extracorporeal life support organization registry international report 2016. Asaio J. 2017;63(4):456-63. https://doi.org/10.1097/ MAT.0000000000000603.

26. Zabrocki LA, Brogan TV, Statler KD, Poss WB, Rollins MD, Bratton SL. Extracorporeal membrane oxygenation for pediatric respiratory failure: Survival and predictors of mortality. Crit Care Med. 2011;39(2):364-70. https://doi.org/ 10.1097/CCM.0b013e3181fb7b35. 